\title{
Circuit
}

Musiques contemporaines

\section{ECM+ Génération 2014 : regards sur les quatre compositeurs lauréats ( ${ }^{\text {re }}$ partie)}

\section{Symon Henry}

Volume 25, numéro 1, 2015

URI : https://id.erudit.org/iderudit/1029482ar

DOI : https://doi.org/10.7202/1029482ar

Aller au sommaire du numéro

Éditeur(s)

Les Presses de l’Université de Montréal

ISSN

1183-1693 (imprimé)

1488-9692 (numérique)

Découvrir la revue

Citer ce document

Henry, S. (2015). ECM+ Génération 2014 : regards sur les quatre compositeurs lauréats ( $1^{\text {re }}$ partie). Circuit, 25(1), 86-93. https://doi.org/10.7202/1029482ar d'utilisation que vous pouvez consulter en ligne.

https://apropos.erudit.org/fr/usagers/politique-dutilisation/ 


\section{ECM+ Génération 2014: regards sur les quatre compositeurs lauréats ( $1^{\text {re }}$ partie)}

Symon Henry

Louis Dufort, Paul Frehner, Gordon Fitzell, Nicolas Gilbert, Jean-François Laporte, Nicole Lizée, Cassandra Miller, Ana Sokolović... autant de compositeurs canadiens aujourd'hui bien établis qui ont tous été révélés par l'entremise du concours pancanadien bisannuel de composition Génération de l'Ensemble contemporain de Montréal (ECM+) dirigé par Véronique Lacroix ${ }^{1}$. Venant de célébrer son $20^{\mathrm{e}}$ anniversaire, ce concours - nommé à l'origine Ateliers et Concert - a d'abord rassemblé et dynamisé les forces vives des jeunes compositeurs du Québec seulement. Rebaptisé Génération, il s'est ensuite étendu à celles de l'ensemble du pays à partir de 2000 . Au fil des ans, il a laissé des traces profondes modelant le paysage de la relève canadienne et contribuant à faire connaître la musique de 53 jeunes compositeurs ${ }^{2}$ par l'entremise d'un large réseau de collaborateurs et diffuseurs à travers le Canada?.

La formule de ce concours comporte trois phases. Tout d'abord, un jury spécialisé effectue la sélection de quatre compositeurs canadiens de 35 ans et moins, recrutés aux quatre coins du pays. La seconde phase consiste ensuite en la présentation, quelques mois plus tard, d'Ateliers et mini-concerts lors desquels une œuvre du corpus de chacun des lauréats est interprétée devant public, en plus d'esquisses en vue d'une composition sur mesure pour l'ensemble qui sont tra- vaillées et explorées directement avec les musiciens. Finalement, le concours se clôt par la tenue d'une tournée pancanadienne de six à neuf concerts mettant en vedette les créations achevées des lauréats.

Étant donné la rareté de l'opportunité que ce projet offre aux compositeurs d'ici, ainsi que la longévité et la notoriété qu'il a acquises au fil du temps, il nous semble pertinent d'étudier les démarches des compositeurs qui ont participé à l'édition 2014 de Génération, soit Marie-Pierre Brasset (1981, Québec), Alec Hall (1985, Ontario/New York), Evelin Ramon (1979, Québec/La Havane) et Anthony Tan (1978, Alberta/ Berlin), afin de présenter certaines préoccupations qui les habitent. Ce travail permettra d'ébaucher des pistes d'analyse basées à la fois sur l'étude de plusieurs partitions de chaque compositeur, sur des entretiens privés $^{4}$ que nous avons eus avec chacun d'entre eux ainsi que sur le matériel ${ }^{5}$ présenté lors des Ateliers et mini-concerts de mars 2014. Considérant le cadre du présent article, le travail de chaque compositeur sera abordé par un seul axe d'analyse pour chacun: la notion de timbre comme expression personnelle chez Evelin Ramon; l'idée de musique en tant que «situation» chez Anthony Tan; l'appropriation de traditions musicales et des sonorités de territoires chez Marie-Pierre Brasset et le rapport à la figuration sonore chez Alec Hall. 


\section{Evelin Ramon : du timbre comme expression personnelle}

Ce que je cherche, ce sont de nouveaux moyens techniques qui puissent se prêter à n'importe quelle expression de la pensée et la soutenir.

\section{- Edgard Varèse}

Cette citation, placée en exergue d'Accumulations (orchestre à vent, 2011) d'Evelin Ramon ${ }^{6}$, illustre particulièrement bien le travail récent de la compositrice. Ayant émigré de La Havane (Cuba) vers Montréal en 2008, elle a depuis développé un grand intérêt pour l'exploration et la personnalisation de modes de jeux alternatifs. Ceci coïncide d'ailleurs avec sa découverte des écrits de Pierre Schaeffer ${ }^{7}$ et de Stéphane Roy ${ }^{8}$ ainsi que de la conceptualisation en unités sémiotiques temporelles ${ }^{9}$ telle qu'elle est développée par le laboratoire Musique et Informatique de Marseille.

Grâce à ces découvertes, elle a mis au jour une des lignes de force majeures de son travail actuel: écrire une musique d'expression éminemment personnelle par l'appropriation d'éléments timbraux expérimentaux. Les écrits précédemment mentionnés lui ont permis d'aborder le timbre comme élément structurant de sa manière de composer. La pièce interprétée lors des Ateliers et mini-concerts de l'ECM+, Analogies (2012), marque d'ailleurs un tournant majeur dans la production de Ramon. Cette pièce représente pour elle le début d'une écriture qu'on pourrait qualifier de «musique concrète instrumentale », si l'expression n'avait pas déjà été fortement associée au travail du compositeur allemand Helmut Lachenmann. Si ce dernier a voulu subvertir l'appareil culturel instrumental occidental par ses explorations structuralistes, Ramon, pour sa part, cherche plutôt à puiser, du côté des outils d'analyse électroacoustique et de l'exploration bruitiste de modes de jeux alternatifs, de nouvelles manières de penser la composition. Ainsi, Analogies peut être entendue comme une illustration instrumentale convaincante de l'introduction du texte fondateur de l'analyse électroacoustique de Denis Smalley, La spectromorphologie: une explication des formes du son:

Lart de la musique ne se contente plus désormais d'emprunter ses modèles sonores aux instruments et aux voix. [...] Pour les auditeurs, les liens traditionnels avec la production physique du son se trouvent fréquemment rompus: souvent, les formes sonores et les propriétés électroacoustiques n'indiquent pas leurs sources ni leurs causes. C'en est fini des articulations familières des instruments et de l'expression vocale; c'en est fini de la stabilité de la note et de l'intervalle; c'en est fini aussi de la référence au temps et à la mesure. [...] Les deux parties du mot [spectromorphologie] renvoient à l'interaction entre les spectres sonores (spectro) et les façons dont ils changent et se configurent à travers le temps (morphologie). La spectro- ne peut exister sans la -morphologie et vice versa: quelque chose doit recevoir une forme, et cette forme doit avoir un contenu sonore ${ }^{10}$.

Le premier mouvement de l'œuvre «Relinquere ", de quelque deux minutes, est un exemple éloquent de ces idées. Un comportement global simple est à la base de son écriture: un grand processus d'accumulation suivi d'un bref filtrage est précédé de deux impulsions en guise d'introduction (figure 1).

Ce processus est articulé, de l'intérieur, par la superposition de trois trames. La première est exclusivement centrée autour du jeu de tambour à ressort (spring drum). Au cours des trois parties, cette couche sonore

FIGURE 1 Evelin Ramon, Analogies (2012), $1^{\mathrm{er}}$ mouvement, "Relinquere", forme d'onde du mouvement complet.

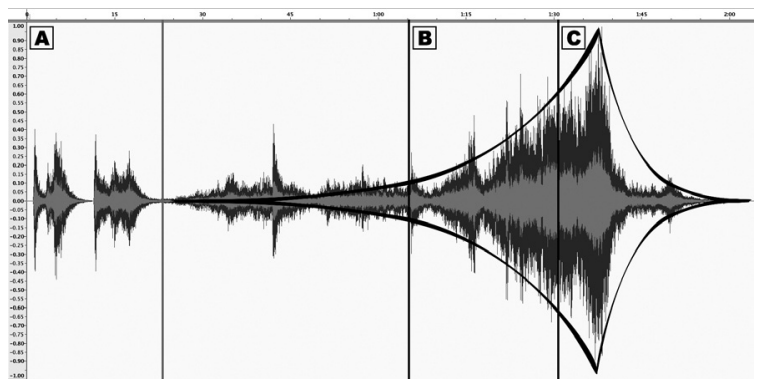


sera essentiellement construite autour de crescendos et decrescendos articulés à l'aide de différents modes de jeux: en frottant une règle de plastique sur le ressort, en secouant l'instrument ou, plus rarement, en frottant rapidement un ongle sur le ressort de haut en bas. À cette première trame s'ajoute une autre centrée sur le jeu de la flûte basse et de la clarinette. Dans la première partie du mouvement (partie A, mes. 1 à 19), elles ne ponctueront qu'épisodiquement le discours de slap tongue (voir figure 2). Cette trame d'impulsions ponctuelles cédera sa place à une trame continue mêlant hauteurs déterminées et voix des interprètes, créant du coup une trame mélodique bruitée (partie B, mes. 20 à 27). La partie $\mathrm{C}$ (mes. 28 à 36) sera à nouveau essentiellement continue, d'abord en superposition de multiphoniques (mélange de bruit et de hauteurs définies) devenant trilles (hauteurs définies uniquement), puis, après le sommet, uniquement des sons d'air (bruit seul).

La dernière trame, aux cordes, est la plus directionnelle des trois. Basée sur la séparation entre les actions de la main droite et de la main gauche, elle ne va que dans le sens du crescendo et de la complexification jusqu'au sommet du mouvement. Au début de celui-ci, les instrumentistes effectuent un trémolo des doigts de la main gauche sur les cordes, heurtant aussi rapidement que possible des hauteurs volontairement imprécises autour de la première position. Au cours de la partie $\mathrm{A}$, l'archet ne servira qu'à partir de la mes. 12 à faire quelques tenues extreme sul ponticello. Dans la partie B, l'archet servira à articuler une trame secondaire de balayage des cordes, en cinq noires contre quatre par mesure (tout le mouvement est en 4/4), de l'aigu au grave et vice-versa. Dans la partie C, ce mouvement s'accélère pour devenir un balayage en triples croches.

Ce type de travail est à la base des deux autres mouvements d'Analogies, mais aussi de son récent qua- des esquisses de sa pièce pour la tournée Génération. Une écriture, donc, qui se souvient de ses origines instrumentales, mais qui tente de dépasser les limites physiques archétypales des instruments ou encore de teinter d'un travail timbral important certains éléments hérités de la tradition classique européenne. Il se dégage donc de ses pièces une impression de distance face à la tradition, mais ce recul, contrairement à un travail intellectuellement critique ou postmoderne, est plutôt une manière de prendre possession d'éléments de cet univers culturel afin d'exprimer des émotions viscérales.

\section{Anthony Tan : musique en tant que situation}

Il nous faudra donc former des images du genre de celles qui peuvent être conservées très longtemps en mémoire. Ce sera le cas si nous établissons des similitudes aussi frappantes que possible; si nous employons des images qui ne soient ni muettes ni floues mais qui soient en action; si nous leur conférons une beauté exceptionnelle ou une laideur singulière; si nous en embellissons, par exemple avec des couronnes ou des habits de pourpre, pour que nous retenions mieux la ressemblance; si nous enlaidissons un objet que nous présenterons par exemple souillé de sang ou de boue ou barbouillé de rouge pour que l'aspect soit plus caractéristique; ou si nous donnons à ces images quelques traits amusants; ce moyen aussi permettra de conserver plus facilement le souvenir ${ }^{11}$.

Pour sa part, Anthony Tan ${ }^{12}$ place, en exergue de l'une de ses pièces (Pose II: On the Shadows of Ideas, pour piano et live-electronics, 2013), un extrait de La rhétorique à Herennius, ouvrage du $\mathrm{I}^{\mathrm{er}}$ s. av. J.-C. jadis attribué à Cicéron mais aujourd'hui considéré d'auteur inconnu. Ces images dont l'auteur parle, Tan cherche à les représenter sous la forme de "poses ${ }^{13} »$ musicales. Une quête de «l'arrêt sur image » dans un art du temps qui passe est donc sous-jacente à son travail des dernières années.

Observing the Ph(r)ase (octuor instrumental, 2013) est pour nous emblématique de cette idée. L'œuvre 
FIGURE 2 Evelin Ramon, Analogies (2012), $1^{\text {er }}$ mouvement, "Relinquere", évolution de la superposition de trames dans les parties A (ex.: mes. 11-14), B (ex.: mes. 23-24) et C (ex. : mes. 30).
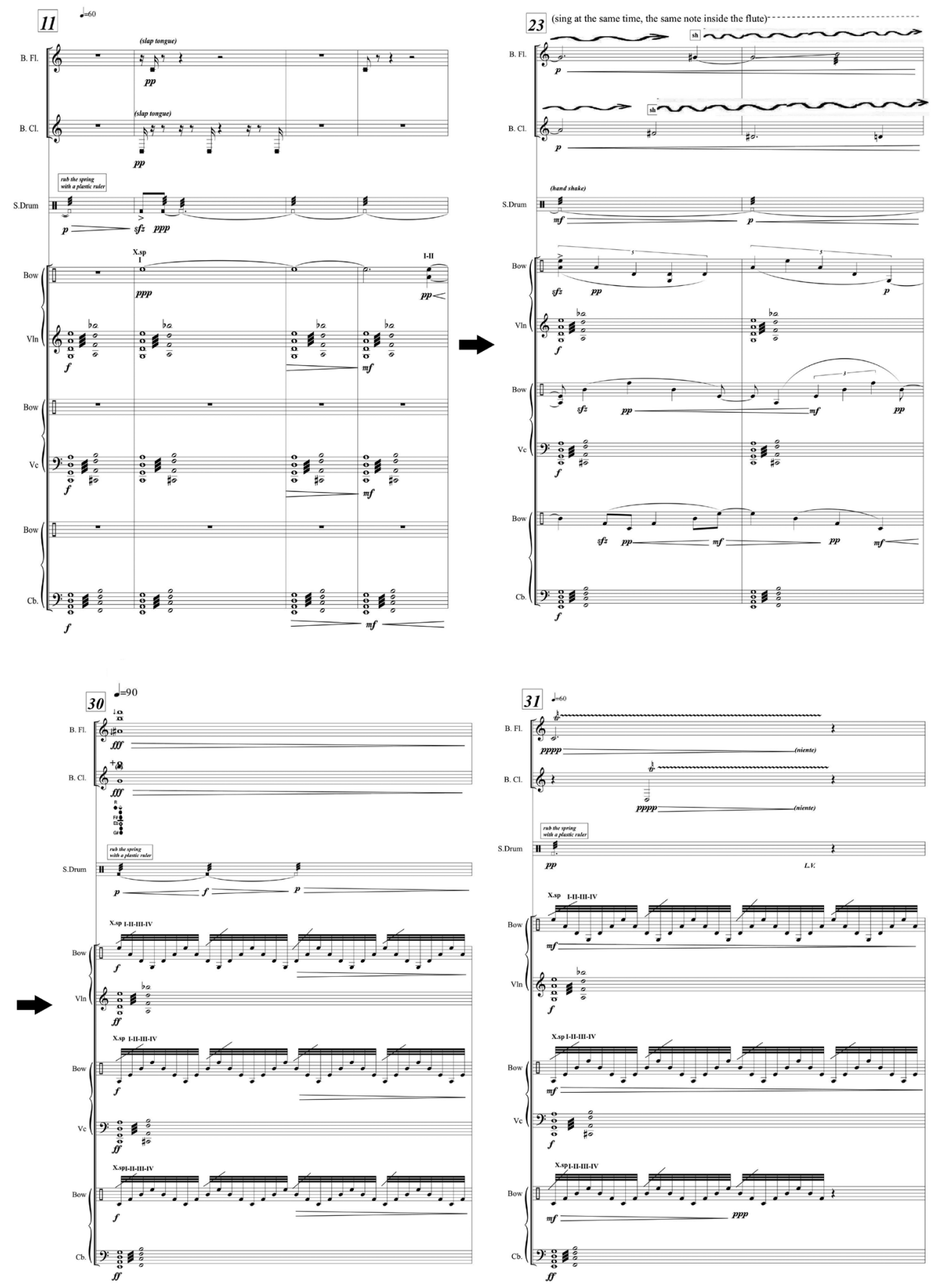
FIGURE 3 Anthony Tan, Observing the Ph(r)ase (2013), structure rythmique de base ${ }^{14}$.

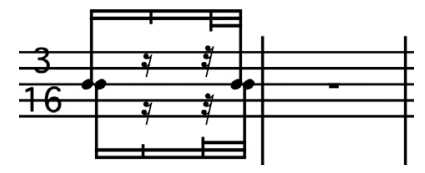

est fondée sur la structure rythmique suivante (voir figure 3), énoncée sous forme d'ostinato à la grosse caisse dans la section centrale (mes. 196 à 234) de la quatrième et dernière partie de l'œuvre.

Cet élément ( $\mathbf{r}$ et ses dérivés $\mathbf{r} \mathbf{1}, \mathbf{r} 2$, etc.; voir figure 4) n'est autre que l'enchaînement de deux structures «impulsions/espace de résonance» de durées similaires: cinq, puis sept triples croches. Il articule, entre autres, l'entièreté de la première sous-section de l'œuvre (mes. 1-16).

Dans cet exemple, nous pouvons remarquer que la cellule $\mathbf{r}$ mène systématiquement à des espaces de résonances composées, chronométrés de 4 à 10 secondes, puis à une terminaison ou encore à un enchaînement avec la forme suivante de $\mathbf{r}$. Il y a aussi ici, en germe, une des tensions contradictoires fondamentales au travail de Tan. En effet, par son intérêt pour la notion de «pose », le compositeur cherche à s'éloigner d'une certaine idée de téléologie pour plutôt s’approcher

FIGURE 4 Anthony Tan, Observing the Ph(r)ase (2013), mes. 1-16.

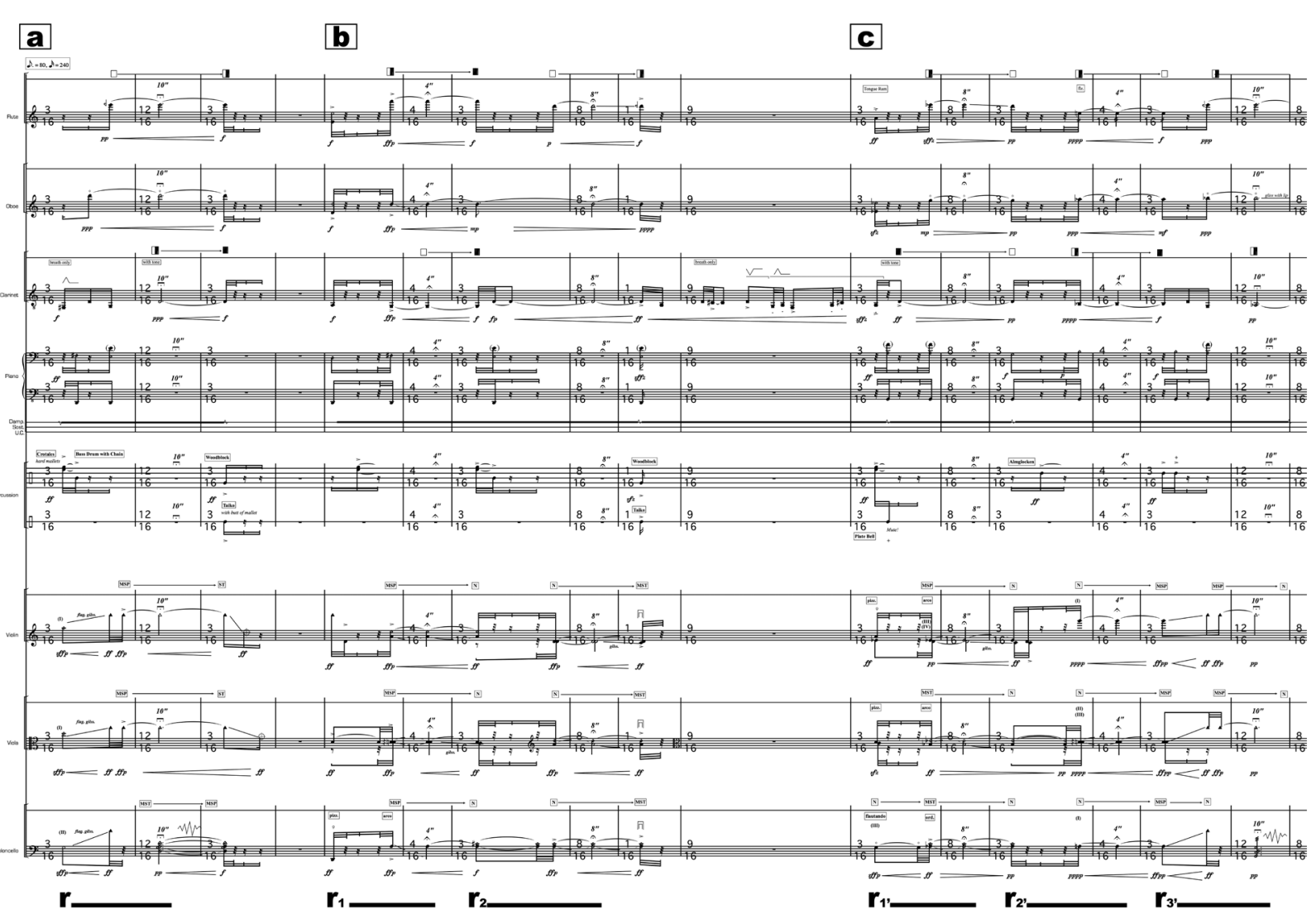




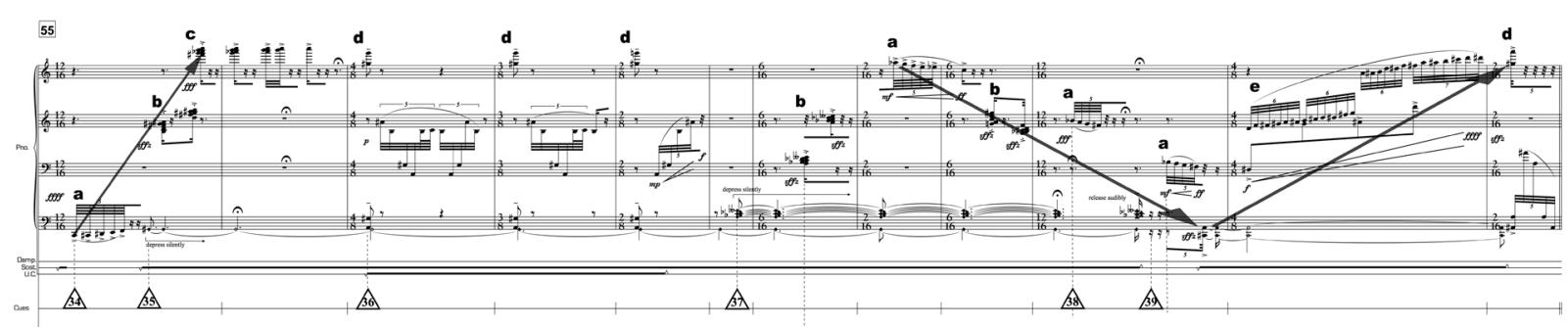

d'une idée de musique en tant que situation proche des conceptions d'Helmut Lachenmann ${ }^{15}$. Il dispose donc dans le temps ses structures sonores, ici délimitées par des silences, en jouant sur un ensemble de caractères similaires afin de susciter chez l'auditeur une écoute mnémonique. La situation de cette première sous-section en est donc une où trois incarnations d'une même identité structurelle sont présentées avec une volonté de non-hiérarchisation: les nuances globales sont similaires, les durées des points d'orgue chronométriques sont organisées symétriquement (10-4-8 | 8-4-10 secondes) plutôt que de manière croissante ou décroissante, les densités, niveaux de complexité, structures rythmiques de base et orchestration sont aussi, finalement, similaires. Pourtant, un élément téléologique majeur subsiste: le segment a est composé d'une seule cellule $\mathbf{r}$, puis le segment $\underline{b}$, après un silence de trois doubles croches, est composé de deux cellules $\mathbf{r}$, le segment $\underline{c}$, finalement, cette fois après un élément soliste à la clarinette basse de neuf doubles croches, est composé d'un enchaînement de trois cellules r. Cette structure formelle basée sur l'idée de développement porte clairement la première sous-section vers la suite de l'œuvre.

Dans Pose II: On the Shadows of Ideas (piano et électronique optionnelle, 2012-2013), cette tension entre «pose» et mouvement est d'autant plus évidente par la nature des objets musicaux choisis par le compositeur. Dans la figure 5, présentant un segment typique de l'œuvre, on peut remarquer une structure construite à partir d'un montage de cinq objets musicaux fortement typés (identifiés par les lettres a à e). La structure globale est d'abord directionnelle (mes. 55) par la répartition des objets a à $\mathbf{c}$ de l'extrême grave de l'instrument à son extrême aigu. Vient ensuite un enchaînement de trois objets $\mathrm{d}$ : leur séquençage ainsi que leur nature même (disposition aux registres graves/médiums/aigus et organisation motivique en mouvements de va-et-vient à l'intérieur d'un ambitus limité) sont l'antithèse de la téléologie de la mes. 55 . Le segment se termine par un montage d'objets $\mathbf{a}$ et $\mathbf{b}$ allant de l'aigu au grave, puis par ce que Messiaen aurait appelé un «groupe-fusée » (objet e) menant à une dernière instance de l'objet $\mathbf{d}$.

Là où les "poses » d'Observing the $\mathrm{Ph}(r)$ ase étaient organisées entres elles de manière finalement directionnelle, les objets de Pose II : On the Shadows of Ideas nous apparaissent comme encore plus marqués par la dialectique «immobilité/mouvement » : s'ils semblent, dans la figure 5 , particulièrement empreints d'un sens clair de la direction, c'est sur le plan de la grande forme qu'on remarque plus particulièrement l'immobilité, la succession de poses, de l'œuvre. En effet, si l'on jette un œil à la forme d'onde de la première moitié de la pièce (figure 6), force est de constater - et particulièrement en contraste avec la forme d'onde du $1^{\mathrm{er}}$ mouvement d'Analogies ("Relinquere») d'Evelin Ramon, présentée plus haut - que cette œuvre met en 

(2012-2013), forme d'onde de la première moitié de l'œuvre.

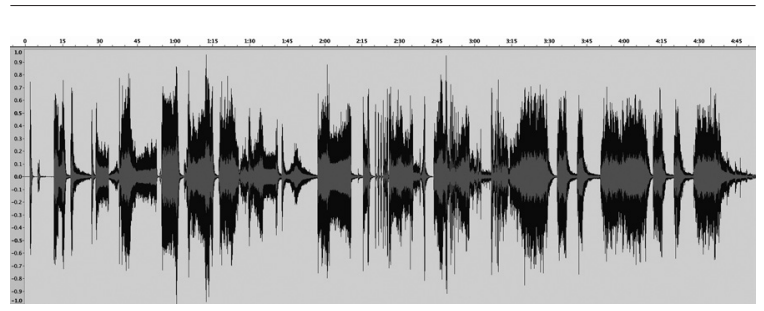

valeur l'absence de sommets clairs ainsi que l'écriture par fragments, deux aspects qui nous semblent particulièrement emblématiques de l'idée de succession de «poses» telle qu'imaginée par Anthony Tan.

La suite dans le prochain numéro de Circuit (vol. 25, $\left.n^{0} 2\right)$.

1. À propos de l'ECM+, voir son site web: <www.ecm.qc.ca > (consulté le 2 février 2015).

2. Voir la liste complète des compositeurs en annexe.

3. Depuis 2000, l'ECM+ a effectué huit tournées pancanadiennes, pour un total de 50 concerts Génération présentés dans un réseau de 14 villes différentes.

4. Entretiens accordés à Symon Henry par Marie-Pierre Brasset (20 mars 2014), Alec Hall (7 mars 2014), Evelin Ramon (24 mars 2014) et Anthony Tan (5 mars 2014).

5. En quelque deux heures consacrées à chaque participant, le public était d'abord invité à assister à un échange entre le compositeur Nicolas Gilbert et le lauréat, au cours duquel la démarche de ce dernier était présentée de manière générale. Une œuvre de musique de chambre de son corpus était ensuite interprétée. Puis, en ayant pour base un Cahier des compositeurs remis au public et contenant des partitions et indications textuelles d'expérimentations sonores, les musiciens de l'ECM + ainsi que sa directrice artistique et chef, Véronique Lacroix, ont travaillé et remis en question les esquisses de leur œuvre en gestation.
6. À propos d'Evelin Ramon, voir son site web: $<$ http://evelineramon.wix.com/evelinramon> (consulté le 2 février 2015).

7. Pierre Schaeffer (1966), Traité des objets musicaux: essai interdisciplines, Paris, Seuil.

8. Stéphane Roy (2000), L'analyse des musiques électroacoustiques: modèles et propositions, Paris, L'Harmattan.

9. "Les Unités Sémiotiques Temporelles sont des fragments sonores qui, même hors de leur contexte musical, possèdent une signification temporelle due à leur organisation." Jean Favory (2007), "Les unités sémiotiques temporelles", Math. Sci. Hum Mathematics and Social Sciences, $45^{e}$ année, $n^{\circ} 178$, p. 51.

10. Denis Smalley (1999), "La spectromorphologie: une explication des formes du son ", Ars Sonora, n ${ }^{\circ} 8,<w w w$.ars-sonora.org/html/ numeros/numeroo8/08d.htm> (consulté le 2 février 2015).

11. Auteur inconnu (2003), La rhétorique à Herennius, texte établi et traduit par Guy Achard, Paris, Les Belles Lettres, p. 122.

12. À propos d'Anthony Tan, voir son site web:

$<$ http://anthonytanmusic.com> (consulté le 2 février 2015).

13. "J'ai essayé de traduire en musique les postures statiques trouvées dans les arts plastiques et visuels afin d'explorer une autre dimension de la notion de geste qui imprègne le discours musical actuel. Mon travail a été inspiré par les esquisses des artistes de la Renaissance, qui, bien que fragmentaires, contenaient déjà l'essence de l'œuvre complète à suivre." Extrait de la note de programme de Trois esquisses sur une pose (pour quatuor instrumental et électronique, 2009, rév. 2013).

14. Les extraits de partitions d'Anthony Tan son reproduits ici avec la permission des éditions Gravis Verlag GmbH, Brühl (Allemagne), 2014.

15. "[...] certaines œuvres musicales peuvent être considérées comme un texte, c'est-à-dire comme un langage. Boulez, par exemple, utilise des titres comme commentaire ou clause: tous ces mots viennent de la littérature. Une phrase est également une partie d'un texte, du langage. Et pour moi, ou bien la musique c'est cela, ou bien c'est une situation, ce qui est complètement différent. Quand je parle de musique en tant que situation, je veux dire par là une situation auditive ou acoustique." Helmut Lachenmann, cité in Abigail Heathcote (2007), "De la musique comme situation: entretien avec Helmut Lachenmann", Circuit, musiques contemporaines, vol. 17, $n^{\circ} 1$, p. 81. 
ANNEXE Compositeurs ayant participé au projet Génération depuis ses débuts.

\begin{tabular}{l|l|l|}
\hline \multicolumn{2}{|c|}{ Compositeurs de toutes les provinces canadiennes (depuis 2000) } \\
\hline 2014 & Marie-Pierre Brasset (QC), Alec Hall (Ont.), Evelin Ramon (QC), Anthony Tan (Alb.) \\
\hline 2012 & Annesley Black (Ont.), Gabriel Dharmoo (QC), Marielle Groven (N.-É.), Riho Esko Maimets (Ont.) \\
\hline 2010 & Simon Martin (QC), Christopher Mayo (Ont.), Cassandra Miller (C.-B.), Gordon Williamson (Ont.) \\
\hline 2008 & Michael Berger (Alb.), Scott Good (Ont.), Brian Harman (Ont.), Fuhong Shi (Ont./Chine) \\
\hline 2006 & Charles-Antoine Fréchette (QC), Aaron Gervais (Alb.), David Litke (C.-B.), Maxime McKinley (QC) \\
\hline 2004 & Wolf Edwards (C.-B.), Nicolas Gilbert (QC), Vincent-Olivier Gagnon (QC), D. Andrew Stewart (Ont.) \\
\hline 2002 & Louis Dufort (QC), Nicole Lizée (Man./QC), Marci Rabe (C.-B.), Patrick Saint-Denis (QC) \\
\hline 2000 & Rose Bolton (Ont.), Emily Doolittle (N.-É.), Gordon Fitzell (C.-B.), Jean-François Laporte (QC), Andriy Talpach (Alb.) \\
\hline Compositeurs exclusivement québécois (de 1994 à 1999) \\
\hline 1999 & Alain Beauchesne, Vincent Collard, Patrice Coulombe, Justin Mariner \\
\hline 1998 & Paul Frehner, Jean-François Laporte, André Ristic, Francis Ubertelli \\
\hline 1996 & Bernard Falaise, Sean Ferguson, Michel Frigon, Ana Sokolović \\
\hline 1995 & Pierre Klanac, Silvio Palmieri, Marc Hyland, Danielle P.-Roger, Daniel Tremblay \\
\hline 1994 & Suzanne Hébert-Tremblay, Estelle Lemire, Isabelle Marcoux, Jacques Tremblay \\
\hline
\end{tabular}

